Meta

Journal des traducteurs

Translators' Journal

\title{
La traduction littéraire est-elle un genre à part ?
}

\section{Jean-Paul Vinay}

Volume 14, numéro 1, mars 1969

URI : https://id.erudit.org/iderudit/004570ar

DOI : https://doi.org/10.7202/004570ar

Aller au sommaire du numéro

Éditeur(s)

Les Presses de l'Université de Montréal

ISSN

0026-0452 (imprimé)

1492-1421 (numérique)

Découvrir la revue

Citer cet article

Vinay, J.-P. (1969). La traduction littéraire est-elle un genre à part ? Meta, 14(1),

5-21. https://doi.org/10.7202/004570ar d'utilisation que vous pouvez consulter en ligne.

https://apropos.erudit.org/fr/usagers/politique-dutilisation/ 


\section{la traduction littéraire est-elle un genre à part?}
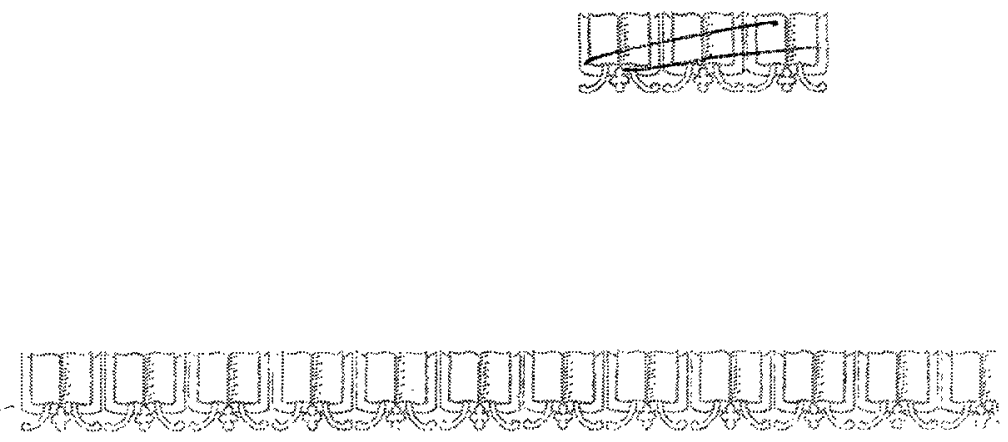

"Unlike the members of most other professions, the literary translator has no examination to pass and no specialized training to undergo; again unlike the members of most other professions, he has to know his job. "

1 (1) Josef ŠKoreckY̌, Babel, XIV, 2 (1968).

dans lesquelles la discussion peut s'engager.

Comme le dit très bien G. Mounin, tous les arguments contre la traduction se résument à un seul: elle n'est pas l'original. On peut, cependant, par des approximations successives, s'approcher de l'original, et cela à tous les niveaux: sémantique, stylistique, culturel, esthétique, personnel. Ces approximations ne

1. Pour alléger l'exposé, plutôt que pour céder à une mode récente des pseudo-mathématiciens, j'utiliserai certaines abréviations relativement simples et transparentes, dont voici la liste:

LD : - Eangue de départ (Source language).

LA: - . L Langue d'arrivée (Target language).

TG - Traduction générale, c'est-à-dire non littéraire, ce terme pouvant être précisé comme suit:

TG-Off. = Traduction de textes officiels, gouvernementaux, etc.

TG-Tech. = Traduction de textes techniques.

TG-Publ. = Traduction de textes publicitaires, etc.

TL $=$ Traduction littéraire.

SCFA = Stylistique comparée du français et de l'anglais (ouvrage de Jean-Paul Vinay et Jean Darbelnet).

F propos de ces abréviations, je ne puis m'empêcher d'avouer que je préfère langue d'arrivée et langue de départ à "langue-source ", "langage source ", "langue-cible " et "langage cible " ou "langage objet que ces mêmes pseudo-mathématiciens affectionnent, pour en avoir attrapé le virus aux U.S.A., comme dit Larousse. Je trouve d'ailleurs avec plaisir les termes LD et LA employés (sans guillemets) par les spécialistes de la traduction qui ont rédigé le compte rendu des séances du congrès de Lahti (Babel, XII, 1 (1967):6). Toujours pour alléger l'exposé, j'utiliserai quelques notes infra-paginales bien senties qui, contrairement aux habitudes reçues, devraient être méditées comme un accompagnement discret, dans la marge ou dans la coulisse, du corps du texte. Cette habitude me vient sans doute de la lecture des ouvrages allemands, dont on dit que "They cannot take a step without leaving a footnote». 
devraient pas nous dispenser de lire l'original, mais ce n'est malheureusement pas toujours possible et la traduction demeure un mal nécessaire, voire indispensable. Le fait qu'il existe des traducteurs depuis la plus haute antiquité devrait nous rassurer là-dessus; et puisque traduction il $\mathrm{y} a$, un bref rappel historique pourrait éclairer la lanterne des traducteurs (et des lecteurs) modernes qui ne songent peut-être pas assez que leurs problèmes ont été aussi ceux de leurs devanciers. La perspective historique est d'ailleurs riche d'enseignement en matière de traduction, comme l'ont bien montré les grands auteurs en la matière: E. Cary, G. Mounin, T. Savory, V. Larbaud et bien d'autres. Un cours de traduction diachronique me paraît donc indispensable à la bonne formation des jeunes traducteurs - qu'on se rassure, je ne ferai qu'en évoquer ici la possibilité.

Je songe notamment à l'étude systématique des traductions successives d'un même texte, par exemple, la Bible ou le Nouveau Testament, qui essaient de transmettre aussi fidèlement que possible le même message, mais qui ont été constamment revues, corrigées, modifiées

a) pour serrer de plus près le sens de l'original, que des recherches concurrentes dans le domaine de l'exégèse et de l'histoire permettent de mieux comprendre;

b) pour aligner ces textes sur les canons esthétiques et stylistiques de chaque époque, cette deuxième considération comprenant les changements grammaticaux et sémantiques.

Un rapide examen des missels publiés de 1867 à 1967, pour prendre une tranche commode de 100 ans, ferait ainsi ressortir des changements considérables. En 1867, on pouvait encore trouver des missels qui utilisaient les anciennes graphies tems et trouvoit (pour temps et trouvait), sans compter bon nombre de conventions typographiques (ponctuation, majuscules) qui nous déroutent. Mais le fond même du texte a aussi changé: le Vous est passé au Tu, le Saint-Esprit est devenu l'EspritSaint (mais pas partout), les Hommes de bonne volonté ont complètement disparu, n'en déplaise à Jules Romains ainsi qu'Ainsi-soit-il, faute d'avoir trouvé une bonne traduction ${ }^{2}$.

Ces mêmes changements se retrouvent, sans doute moins sensibles parce que moins familiers, tout au long de l'histoire des grandes traductions. Relisons les éditions successives de Shakespeare, d'Homère ou des Mille et Une Nuits ${ }^{3}$ et nous pourrons nous demander, parfois, si nous lisons bien le même texte. On a souvent l'impression de paraphrases plutôt que de traductions; mais il faut bien dire qu'il

2. C'est là un sujet passionnant, qui devrait inspirer les étudiants en mal de thèse. Les textes traditionnels des Ecritures sont, pour certains au moins, si chargés de résonances affectives qu'il est dangereux d'y porter la hache du modernisme. Ce point a bien été mis en valeur par E. Nida, qui se spécialise comme on le sait dans le domaine de la traduction biblique (voir META, XIII, 3: 159-161) dont il expose les problèmes particuliers dans son nouvel ouvrage: The Theory and Practice of Translation with Special Reference to Bible Translating (American Bible Society, pre-publication edition, 1968). On pourra également consulter l'article d'Aimon-Marie Roguet dans Traduire, 43 (1965) intitulé * Problèmes des traductions liturgiques ». Quant à Jules Romains, et avec lui tous les auteurs anglais et français qui ont donné à leurs ceuvres des titres extraits de citations bibliques ou des Evangiles, il risque fort de voir son titre perdre de sa force stylistique, puisque l'allusion directe sera perdue. L'expression Hommes de bonne volonté s'était d'ailleurs considérablement « laïcisée » et figurait en bonne place dans les discours républicains de nos distributions des prix. Maintenant que la traduction se lit «paix sur la terre aux hommes qu'il (Dieu) aime», les orateurs affranchis feront bien de refourbir leur arsenal de rhétorique.

3. Je recommande aux lecteurs de META les pages denses et très documentées que E. Cary a écrites sur ces problèmes historiques (Voir Bibliographie, 4.1). 
nous manque un élément capital pour étayer notre jugement: nous ignorons la façon dont les lecteurs des différentes époques réagissaient à des textes étrangers. Si $\mathrm{M}^{\text {me }}$ Dacier change ou supprime des détails importants de l'Odyssée, c'est parce qu'au XviII ${ }^{\mathrm{e}}$ siècle, son public s'en effarouchait. Il faut avouer que, sur ce chapitre, nous sommes devenus plus coriaces, et que la scatologie d'un Céline ou l'érotisme d'un Lawrence ne nous effarouchent guère ${ }^{4}$. Mais, pour reprendre l'exemple de Lawrence, puisqu'on en parle, est-on bien sûr de se trouver, en 1968, face à une traduction de l'Amant de Lady Chatterley, dans la situation émotive du lecteur anglais de 1928 qui prenait la mouche pour un rien et n'osait lire les romans français sous prétexte qu'ils étaient imprimés sous couverture jaune?

À ce point de la discussion, on pourrait signaler que la même difficulté fattend le lecteur du $\mathrm{xx}^{\mathrm{e}}$ siècle qui lit des textes anciens rédigés dans sa langue maternelle. Est-on bien sûr de comprendre Racine? Faut-il rire aux plaisanteries de Shakespeare? Nous passons ainsi à côté de beaucoup de difficultés, faute de notes explicatives dans le texte original. J'aime citer à ce propos les deux premiers vers de la première Élégie de John Donne (1573-1631 - comme quoi les dictionnaires ont du bon) que je lisais sans les comprendre, jusqu'au jour où Cazamian m'a fait remarquer qu'au XvI $\mathrm{X}^{\mathrm{e}}$ siècle, l'anglais fond voulait dire «fou, insensé »:

Fond woman, which wouldst have thy husband die,

And yet complain'st of his great jealousie.

2. IiHe heart of the MATter

Ceci dit, venons-en au fond du problème: peut-on parler de TL comme d'une discipline à part, pour laquelle d'ailleurs point ne serait besoin de formation particulière, puisqu'elle semble naître toute armée dans le cerveau des «grands traducteurs »? Peut-on surtout - c'est ce qui m'intéresse au premier chef essayer de systématiser le travail du traducteur en TL comme on a essayé de le faire, même imparfaitement, dans le domaine de la TG ? Ce dernier point a comme corollaire la question de la formation du traducteur littéraire, en dépit des proclamations d'indépendance et de self-made-man-isme (sic). Je pose la question, parce que si la TL n'est pas un genre à part, les principes de la stylistique comparée s'y appliquent comme ailleurs; si c'est un genre à part, il faut essayer de définir en quoi elle diffère de la TG et de ses subdivisions: TG-Off., TG-Tech. et TG-Publ., pour ne citer que celles-là.

Remarquons tout d'abord que, pour beaucoup de traducteurs européens (et assez curieusement américains, mais non canadiens), la TL est la seule traduction digne de ce nom; quand on parle de "grands traducteurs 》, on ne pense jamais, semble-t-il, aux traducteurs gouvernementaux, scientifiques ou publicitaires.

Lorsque le regretté Edmond Cary a composé son étude sur les Grands Traducteurs français, il a tout naturellement songé à des traducteurs littéraires tels Gérard de Nerval, Amyot, Galland, $\mathbf{M}^{\mathrm{me}}$ Dacier, Leconte de Lisle, Georgin; et c'est de TL que traitent tous les manuels qui sondèrent la traduction comme un art. Il semble

4. Ce point soulève d'ailleurs d'autres difficultés, pour les traducteurs qui ont encore un code de moralité et qui prétendent le suivre. Mais après tout, il est toujours possible de refuser un contrat. Mieux vaut ne pas traduire que de vouloir à tout prix expurger et dénaturer. 
qu'on ne puisse exiger «qualité » et «fidélité » que des seuls traducteurs littéraires; on réclame pour eux tous les attributs de l'écrivain: sensibilité, sens de la langue, habileté à manier les ressources stylistiques, culture générale, curiosité intellectuelle, sens du public, etc. On peut évidemment rejeter ces opinions en bloc, et prétendre que la TL est un simple cas particulier de la TG, ce qui est exact sur plus d'un point. Mais il m'a semblé intéressant de rassembler quelques-unes des raisons qui poussent les traducteurs littéraires à adopter cette attitude d'ostracisme vis-à-vis de leurs collègues de la TG, attitude qui ne va pas, il faut l'avouer, sans une bonne dose de condescendance.

Voici plusieurs arguments que je relève dans des publications récentes.

L A TL S'ADRESSE À UN PUBLIC DIFFÉRENT

1 - Par exemple, $\mathrm{M}^{\mathrm{me}} \mathrm{D}$. Olivier (Traduire, 52) réclame l'absence de tout jargon scientifique dans les textes des membres de la SFT, qui sont des «traducteurs littéraires travaillant pour un vaste public». Selon Michel Mohrt, du Figaro littéraire, le nombre des traductions d'ouvrages littéraires étrangers serait devenu, en France, « aussi important que celui des romans français».

\section{La TL TRAVAILle DANS UNE OPTIQUe DIFFÉRENTE}

L'article du Figaro littéraire du 25 septembre 1967 rassure les traducteurs littéraires quant aux possibilités de la «machine à traduire»; celle-ci travaillera essentiellement sur des textes scientifiques «dont la syntaxe est généralement facile. La machine est incapable de rendre un texte littéraire où la langue est considérée pour sa forme même $»$. Comme diraient les traducteurs français: «BULLy for you!»

\section{LA TL RELÈye DE LA CRÉATTON LITTÉRAIRE}

Dans son discours au congrès de Lahti, M. Pierre-François Caillé, président de la FIT et fin théoricien de la traduction, évoque en ces termes la différence entre la TL et la TG-Tech.: "Nous touchons ainsi au double problème de la formation et de la spécialisation, à ce point de divergence entre la traduction littéraire qui, tout en ayant les mêmes lois fondamentales, relève plus du don d'écriture et de l'art [ c'est moi qui souligne], et la traduction scientifique qui, elle, ne peut être efficace qu'en s'appuyant sur une discipline stricte et poussée à fond » (Babel, XIII, 1). C'est dire encore une fois qu'on naît traducteur littéraire et qu'on ne le devient pas.

Cette remarque est en réalité plus qu'une simple boutade. Un secteur important de la TL est en fait obligé - point essentiel — de pousser très loin l'adaptation, pour préserver certaines qualités fondamentales de l'original. C'est certainement le cas de la traduction poétique, qui appellerait à elle seule tout un numéro spécial; c'est aussi le cas de la traduction des pièces de théâtre, domaine par excellence de la langue familière, transparente, «qui passe la rampe ». Le type le plus extrême de ce traducteur pourrait bien être Maurice Clavel (voir Traduire, 51) qui « adapte » des pièces anglaises, espagnoles et italiennes pour la scène parisienne. Celui-ci estime en effet qu'il est bon de savoir la $\mathrm{LD}$, mais point trop parfaitement, « car à force de trop bien saisir les moindres nuances du texte original, on finit 
par se noyer dans le détail $\$^{5}$. Le traducteur littéraire, à ce niveau extrêmement raréfié, n'a besoin que d'une seule qualité - en plus de savoir écrire en français, je suppose: «l'inspiration ». M. Clavel lit la pièce qu'il va traduire jusqu'à presque la connaître par cceur; il « la rumine, s'en imbibe, s'en imprègne... Il en rêve la nuit $\gg$. Au terme de cette période de gestation plus ou moins longue, il referme l'original et se met au travail. Il ne s'y reportera plus que dans la seconde phase, celle $\mathrm{du}$ « repiquage », où il essaie, en quelque sorte, de transformer son adaptation en traduction. Quand on lui pose «la fameuse question de la fidélité [ce problème majeur des traducteurs]", $M$. Clavel répond par un cambronnesque «La fidélité ? Je m'en fous... » Pour lui, la seule fidélité qui compte est celle de « thème à thème »: position parfaitement normale pour un adaptateur qui doit rendre au premier chef l'esprit de l'original, sa démarche particulière et recréer dans la LA les rapports psychologiques que l'auteur souhaitait établir entre acteurs et spectateurs. Nous touchons certainement là une des «limites de la traduction», qui font de la TL un genre à part ${ }^{6}$.

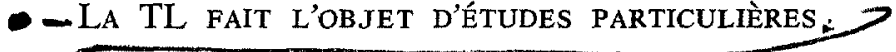

đI est particulierement instructif de consulter sur ce point les principales revues de traducteurs, - sauf apparemment META, qui aborde pour la première fois ce problème. Je consulte actuellement les pages très denses de Traduire, bulletin de la Société française des traducteurs (66, rue Pierre-Charron, Paris $8^{e}$ ) et $\mathbf{j}$ 'y relève, à côté de plusieurs articles scientifiques et techniques, une majorité de communications qui se préoccupent de $\mathrm{TL}$, par exemple « La traduction des livres d'art» $\left(\mathrm{n}^{\circ} 46\right)$; «Problèmes de traductions liturgiques» ( $\left.\mathrm{n}^{\circ} 43\right)$; «Traduction et Cinéma» $\left(\mathbf{n}^{\circ} 25\right)$; «La traduction des noms de personnes [dans les romans] » ( $\left.\mathrm{n}^{\circ} 52\right)$; «La traduction des manuscrits de la Mer Morte $\left(\mathrm{n}^{\circ} 54\right)$; «L'adaptation théâtrale » et «Le problème de l'information dans la traduction des essais » $\left(\mathrm{n}^{\circ} 51\right)$. On relève des articles parallèles dans Informazioni, organe des traducteurs italiens - en particulier dans le numéro 1 (1966), où la TL est longuement étudiée sous la plume de E. Pocar, E. Kalachnikova, E. Esposito, F. Zannino; et naturellement dans Babel, qui ne consacre pas moins de 20 articles à la TL de 1964 à 1967, dont certains très importants: "Poetry and Translation ", de H. W. Chalsma; «Congruence entre l'original et la traduction » de L. Tarnoczi; «La stylistique comparée, base de l'art de traduire» de E. Etkind - sur lequel j'aurai l'occasion de revenir, et même une discussion sur la pertinence du sujet qui nous préoccupe, sous la plume de I. J. Citroen, « The Myth of the Two Professions - Literary and Non-literary Translation ». Enfin, les 264 premières pages

5. Dans le même ordre d'idées, Edmond Cary [Traduire, 47 (1966)] suggère que "l'érudition linguistique mise au service de la traduction moderne apparaîtra peut-être [...] comme la plus monumentale des trahisons envers les poètes du passé ".

6. Je ne puis résister au plaisir de citer ici un extrait de ce même discours de P.-F. Caillé qui brosse avec sa verve habituelle une "image " du traducteur littéraire: "... plongé dans le combat et la vie de la création littéraire, exposé sans cesse à trébucher sur l'interprétation d'un mot, à trahir et son auteur et lui-même, seul et seul juge devant une cuvre qu'il doit faire sienne, repenser, remodeler, revivre, tout en sachant qu'elle n'est pas de lui, exposé, s'il a un talent d'écrivain, à se substituer à un autre, à prendre sa place, comme un coucou de nos forêts s'adjuge le nid d'un autre oiseau - et toujours à chaque page, à chaque ligne, extrayant un mot-clé de son contexte original, le mirant, le soupesant, le comparant pour choisir, dans sa propre langue, son équivalent rythmique, logique, philosophique le plus juste». 
des Actes du $\mathrm{III}^{\mathrm{e}}$ congrès de la FIT traitent avant tout de «la qualité en matière de traduction littéraire ». Il ne m'est pas possible de m'y arrêter davantage (voir Journal des Traducteurs, IX, 1 (1964): 22-24).

\section{La TL Possède une PERSONNalité JuRIDIQUe}

Il existe des groupements spécifiques de spécialistes de la TL; certains congrès se consacrent à leurs problèmes; le sous-titre du Bulletin de la Société française des traducteurs définit la SFT comme étant «le Syndicat national des traducteurs littéraires, techniques, jurés, militaires, de presse, etc. ». On notera la première place donnée à la TL - ainsi que l'absence, en pays monolingue officiellement, de traducteurs gouvernementaux et publicitaires (à moins qu'ils ne soient compris dans la rubrique « technique $»)$.

\section{LA TL, COMME L'ORIGINAL, ABOUTIT À UNE cUVRE D'ART}

C'est sans doute là l'argument principal des tenants de la théorie « La traduction est un art ». Je voudrais verser à ce dossier une citation caractéristique relevée dans la Qualité en matière de traduction, sous la plume d'Armand Pierhal, écrivain et traducteur (p. 97-98):

On doit entendre par qualité d'une TL les mêmes critères qui conditionnent la qualité d'une œuvre littéraire originale, avec cette condition supplémentaire qui est la fidélité du traducteur à la pensée de l'auteur qu'il interprète... Quant à la difficulté de la tâche qui se pose au traducteur littéraire, j'ai plusieurs fois soutenu la thèse suivante, malgré son aspect de paradoxe: quand une œuvre traduite est une auvre d'art, elle est supérieure à l'original.

\section{TRADUCTIONS «PERSONNALISÉES 》 ET TRADUCTIONS IMPERSONNELles}

L'une des différences les plus notables entre TL et TG repose sur l'importance donnée à la personnalité du traducteur, par rapport à cel'e de l'auteur. Il semble évident qu'un texte de loi, par exemple, ou un rapport technique sur la conservation des grains, ou encore la posologie d'un médicament sur un flacon ne reflétent guère, dans leur rédaction originale, la personnalité des auteurs. Il existe même une forte tradition à la fois juridique et scientifique, qui tend à imposer des règles de rédaction ( constraints 》) dépersonnalisant ces textes par la suppression de certaines marques stylistiques — on pense à l'emploi de $\mathrm{Je} / \mathrm{Nous}$-, ou même grâce au recours à des formes syntaxiques particulières qui seraient inadmissibles dans un texte littéraire, par exemple les inversions passives du type: 1) sont déclarés admissibles les candidats suivants, ou les phrases nominales utilisées pour les affiches et panneaux de signalisation: 2) défense de déposer des ordures ou des formules impersonnelles, comme celle que j'ai relevée sur un paquet expédié par la poste: 3) peut être ouvert d'office.

Dans tous ces cas, la traduction ne reflète aucunement la personnalité de l'auteur. No dumping rend exactement la phrase 2 ci-dessus, et postmaster: this parcel may be opened for inspection la phrase 3. Toute infraction aux règles de la stylistique officielle fera par contre un effet certain sur le public averti et pourrait bien refléter la personnalité du traducteur, ou tout au moins son manque de compétence professionnelle. C'est ce qui se passe lorsqu'une marque d'essence m'offre une carte de crédit sous le titre votre passeport pour mieux voyager - 
mais ce n'est pas ici la place d'épiloguer sur cette sorte d'anglicisme à la fois stylistique et culturel qui est sans doute la plus insidieuse et la plus troublante.

Pourtant, en TG-Publ. par exemple, la personnalité de l'auteur d'une affiche ou d'un slogan pourrait fort bien jouer un rôle - et il n'est pas exclu que les affiches de Capiello ou les slogans de certaines officines de Madison Avenue transmettent réellement un message personnel, "signé $X$ », comme on disait avant la guerre. Dans ce cas, le traducteur substitue en partie sa personnalité à celle de son collègue unilingue plus heureux, mais cette substitution s'effectue à l'intérieur de cadres linguistiques et culturels communs à la majorité des locuteurs auxquels s'adresse cette publicité. Ces «contraintes» sont encore très fortes; la personnalité du traducteur, dans la mesure où elle peut s'affirmer, correspond en fait à l'exploitation plus ou moins heureuse de ce qu'on pourrait appeler, d'une manière un peu floue, le «sentiment linguistique » d'une population donnée. Ce même «sentiment »explique pourquoi tel produit anglais se vend grâce au slogan Ssschweppss, en face d'un slogan français du type Dubo - Dubon - Dubonnet.

$\mathrm{Au}$ contraire, et ce contraire est rafraîchissant, le texte littéraire ne saurait exister sans la présence constante et impérative de l'auteur. Cette nouvelle dimension du texte original devra dès lors figurer dans la traduction, et rejoint précisément nos préoccupations de stylisticien (l'écriture). Une de mes étudiantes de Toronto me faisait remarquer que les critiques des TL portent toujours sur la fidélité au sens, sur la félicité de la forme, mais jamais sur la transposition en LA des traits personnels qui caractérisent l'auteur dans le texte LD. Hemingway en français est-il encore Hemingway, ou un simple reflet, en blanc et noir, d'un personnage haut en couleurs?

\section{PERTINENCE D'UNE OPTIQUE STRUCTURALE}

Tout en admettant, devant de tels arguments, que la TL est bien un genre « à part entière », on peut se demander si c'est aussi un domaine privilégié, qui ne connaîtrait d'autres règles canoniques que celles du goût et de l'inspiration. C'est du moins l'impression que laissent la majorité des écrits traitant de TL, et je voudrais prouver que c'est une erreur. Le domaine de la TL est tout aussi structuré que celui de la $\mathrm{TG}$, et d'ailleurs plusieurs traducteurs ont récemment accepté d'analyser les composantes stylistiques et fonctionnelles de leur propre technique. Ils arrivent ainsi à des conclusions proches des nôtres - et la nature humaine étant ce qu'elle est, je tends à leur donner raison. C'est ainsi que je lis dans le récent numéro de Babel (XIV, 2 (1968): 72-73), sous la plume d'Anton Popovic, deux paragraphes très significatifs que je me permets de retranscrire ici dans leur forme originale

A literary translation is not a translation of straightforward subject matter, the mere communication of a neutral semantic substance: it is at the same time a transposition of the total artistic value of the original text... The object of study is the total value of the translated text as a work of art. And what we need is a system with which to determine that value. [...]

[Our] only choice is to compare stylistic values in two different systems of such values.

Ce n'est pas encore très clairement exprimé, mais l'idée est là: quel sera ce 
système qui doit permettre « d'étudier la valeur globale du texte en LA, considéré comme une cuvre d'art »?

\section{LA STYLISTIQUE COMPARÉE D'EFIM ETKIND}

Dans un discours qui a fait beaucoup de bruit, prononcé au congrès de Lahti et reproduit dans Babel, XIII, 1 (1967): 23-30, Efim Etkind, linguiste et traducteur russe s'est penché sur la traduction littéraire, «cette création littéraire au second degré » et propose pour en faire l'analyse d'élargir et même de changer l'acception que l'on donne généralement au terme de stylistique comparée, tel qu'il a été défini par les auteurs des ouvrages de la «Bibliothèque de stylistique comparée » que dirigeait alors (1958) Alfred Malblanc.

Reprochant à ces auteurs (dont je suis) d'identifier la stylistique à la langue dans son ensemble (op. cit., p. 26), cherchant de son côté à «allier indissolublement la linguistique et la théorie de la littérature », M. Etkind propose une stylistique comparée modifiée, aux dimensions très vastes, dont la première partie au moins me semble «traditionnelle», mais qui a le mérite, je dois le reconnaître, d'englober l'analyse des démarches littéraires. Voici un bref résumé du programme en question, qui comporte six « confrontations $»$ ou rapprochements systématiques; savoir

1. la confrontation de deux systèmes linguistiques (structures grammaticales, lexique et phraséologie, etc.);

2. la confrontation des systèmes stylistiques des deux langues;

3. la confrontation des styles littéraires traditionnels dans les deux langues (classicisme, romantisme, etc. et les genres: ode, élégie, fable, etc.);

4. la confrontation des deux systèmes prosodiques dans leur spécificité nationale (jeu des syllabes en français, de l'accent tonique en allemand et en russe, etc.); 5. la confrontation des deux traditions culturelles et historiques des deux civilisations nationales dans la mesure où elles trouvent leur expression dans la tradition littéraire;

6. la confrontation des deux systèmes esthétiques individuels, celui de l'auteur et celui du traducteur.

Ce plan est intéressant, même s'il n'est pas entièrement original. J'ai l'impression en effet que les ouvrages cités à la bibliographie du présent article, notamment notre SCFA, couvrent déjà suffisamment le point (1) - tout en réservant l'avenir, naturellement. Le point (2) est au moins esquissé dans la dernière partie de la SCFA, et découlerait en particulier du rapprochement systématique des ouvrages de stylistique interne du français et de l'anglais ? Il faut souligner

7. En raison de la conjoncture canadienne, c'est évidemment à ces deux langues que nous pensons spontanément; mais ce rapprochement vaudrait pour toute autre paire de langues et même, depuis Barthes et le développement de la stylistique multiple de l'école allemánde, pour tout groupe de langues artificiellement choisi. On pourrait s'acheminer ainsi vers une typologie de la stylistique, vers des Universals of stylistics, pour calquer l'expression de J. Greenberg, vers une stylistique générale en somme, qui serait une branche de l'esthétique, de même que la linguistique générale serait une branche de la sémiologie. Les principes d'une stylistique générale seraient évidemment essentiels pour diriger la recherche en traduction automatique, mais nous n'en sommes pas encore là et les considérations pratiques nous obligent en fait à nous restreindre à l'anglais et au français. 
ici que les stylisticiens anglophones sont très en retard sur leurs collègues francophones, et que les travaux au titre de ce deuxième point n'avanceront guère avant qu'un Bally anglais ou américain ne nous livre le secret des mécanismes * rhétoriques $\gg$ de l'anglais.

Le point 4 est à la mode sur ce continent depuis déjà une dizaine d'années: la phonétique et la phonologie des deux langues sont si bien décrites qu'une «confrontation» systématique est maintenant possible. Il s'agit des travaux dits "différentiels» (en anglais, contrastive) dont on trouvera la bibliographie dans Iral, par exemple. Il faudrait évidemment pousser cette confrontation très loin, sur le plan de la prose littéraire et de la poétique, afin d'étudier les niveaux les plus élevés de la création littéraire.

Le point (5) a été abordé dans la SCFA et repris en détail, notamment par E. Nida; nous sommes là dans le domaine de la métalinguistique, s'il est encore permis d'utiliser cette terminologie. Plusieurs linguistes et anthropologues américains ont consacré depuis quelque temps de nombreux articles et monographies aux effets du choc de deux «cultures». Pour le français, on consultera en particulier les publications de l'école de Seattle.

Restent les point 3 et 6: ce sont ceux-là qui manquent à notre SCFA et qu'il faudra bien abôrder sérieusement, si l'on veut suivre M. Etkind jusqu'au bout. Ce dernier a parfaitement raison de réclamer une confrontation systématique sur les plans du style et des systèmes esthétiques individuels. On peut d'ailleurs se demander pourquoi, au Canada français par exemple, ces deux points n'ont pas été envisagés plus tôt. La raison en est peut-être que, jusqu'à ces derniers temps, il n'y avait pas de débouchés pour la TL à Montréal ou à Québec: toute l'énergie du corps translatif s'épuisait sur des étiquettes de savon en poudre ou s'essoufflait à Ottawa pour rattraper le retard chronique des traductions françaises en matière de publications gouvernementales. On n'avait guère le temps, dans ces conditions, de faire de la littérature et je suis certain qu'une statistique (que je ne puis développer ici) montrerait que ce sont surtout les Anglo-Canadiens qui ont œuvré dans le domaine de la TL, pour faire connaître à leurs compatriotes la production poétique et romanesque du Canada français.

\section{ESQUISSE D'UNE RÉPONSE À M. ETKIND}

Fortifié par les encouragements de cet auteur qui ne semble d'ailleurs pas connaître les travaux de l'école montréalaise ${ }^{8}$, et suivant en cela mon propre enseignement, fondé depuis 1958 dans le cadre du cours de stylistique comparée, je voudrais indiquer succinctement comment on peut concevoir cette liaison indispensable entre le plan linguistique et le plan littéraire.

Pour éclairer le débat, il n'est peut-être pas inutile de rappeler que tout procédé stylistique peut se ramener à l'un des trois types suivants:

8. Il ne cite en effet que les travaux de Malblanc, qui portent sur la comparaison du français et de l'allemand, et pour le seul Whorf, cite abondamment Humboldt, Weisgerber, Seidler, Klemperer. Il ne lit peut-être pas l'anglais. La discussion qui va suivre sur Stylistique-StyleEcriture résume une doctrine élaborée à Edmonton, Montréal et Toronto qui a fait l'objet de plusieurs publications, notamment un exposé dans la Revue de l'Université Laurentienne, où l'on trouvera une bibliographie plus complète. 


\section{1) type universel}

Il appartient à ce qu'Etkind appelle «le système stylistique » d'une langue; un tel procédé est parfaitement repérable et se retrouve chez tous les auteurs qui utilisent une même langue; il est donc universel (à l'intérieur d'une langue donnée).

Soit l'utilisation en français des impersonnels réfléchis: « Le saumon se mange froid ». Cette tournure est commune à tous les usagers du français 1969, de même que la tournure passive «Salmon is eaten cold» est commune aux usagers de l'anglais 1969.

Par contre, l'absence de recours à ce procédé est, soit une faute (anglicisme de syntaxe), soit un tour personnel qui frappe le lecteur: "Le saumon est mangé froid $\gg$ et qui relève alors du type 3 , ci-dessous.

Les ouvrages dits de «stylistique comparée » traitent normalement, sinon exclusivement, de cette sorte de procédés. Leur utilisation correcte caractérise le «bon» écrivain 1969; elle dénote surtout une aisance dans l'emploi de la langue qui est la marque des francophones. Un non-francophone devra se familiariser avec ces procédés et pourra toujours se trahir par une gaucherie dans leur emploi.

\section{2) type localisé}

Un certain nombre de tours et procédés stylistiques relèvent d'un mode ou d'un genre particulier ou encore d'une école littéraire. Ces procédés peuvent, tout en faisant partie du fonds commun de la langue, rester «colorés » par l'emploi qui en a été fait à un certain moment, par certains auteurs. Et cette coloration peut (doit ?) même se retrouver en traduction. Soit le passage suivant:

Et la prudente Pènélopéia, semblable à Artenus ou à Aphroditè d'or, sortit de sa chambre nuptiale. Et les servantes placèrent pour elle, devant le feu, le thrône où elle s'asseyait. Il était d'ivoire et d'argent, et travaillé au tour.

Et c'était l'ouvrier Ikmalios qui l'avait fait autrefois, ainsi qu'un escabeau pour appuyer les pieds de la Reine, et qui était recouvert d'une grande peau. Ce fut là que s'assit la prudente Pènélopéia.

Ce texte est de toute évidence une traduction du grec, avec tous les tours que comporte le genre épique; même l'utilisation de la conjonction et, qui dévie de la norme, nous fait penser au kai homérique; sans compter naturellement les noms eux-mêmes, que l'auteur a conservé avec leur accoutrement antique. parallèle:

Ces remarques deviennent plus pertinentes si nous consultons un texte

Ce prince étoit à peine sorti, que la sage Penelope, semblable à la chaste Diane et à la belle Venus, descend de son appartement suivie de ses femmes, qui lui mettent d'abord près du feu un beau siége fait tout entier d'yvoire \& d'argent, ouvrage d'Icmalius, tourneur célébre, qui y avoit employé tout son art, \& qui y avoit joint un marchepied très-magnifique \& très-commode.

On étendit des peaux sur ce siége \& Penelope s'assit.

Ainsi, à partir d'un même original, deux traducteurs séparés par une centaine d'années, réussissent à nous faire sentir la noblesse du texte par des procédés stylistiques différents, compte tenu des changements de structure de la langue elle-même. 
Il s'agit, on l'aura reconnu, de la traduction de l'Odyssée par Leconte de Lisle et celle, écrite 100 ans auparavant, par $\mathrm{M}^{\mathrm{me}}$ Dacier.

La présence de procédés localisés permet de dire quelle école littéraire l'auteur appartient, dans quel genre et à quelle époque il écrit.

3) type individuel

On peut aller plus lon dans la volé de la déduction et reconnaitre la personnalité de l'auteur, grâce \. tel trait qui lui est particulier ou qui trahit ses préférences, son caractère. Il s'agi (à de l'utilisation individuelle faite par l'auteur de procédés stylistiques universels; cest pour reprendre la phrase de Saussure, la stylistique de la parole (du discours) par epposition à la stylistique de la langue.

Cette triple distinction simplifie forcément et peut-être abusivement la réalité. Il ne sera pas, dans la pratique, toujours facile da dégager ce qui appartient à un type ou un autre, parce qu'il $\mathrm{y}$ a une grande variété do transitions entre les types. Mais cette simplification volontaire est la seule qui permette d'envisager un problème aussi vaste sans tomber dans l'atomisation des observations.

Je propose que, dans un but de clarté, nous appelions \&SFYLTSTIQUE 》 ce qui est universel, «STYLE » ce qui est localisé (après Barthes) ce qui est personnel à un auteur ${ }^{\circ}$

UN EXEMPLE CONCRET:

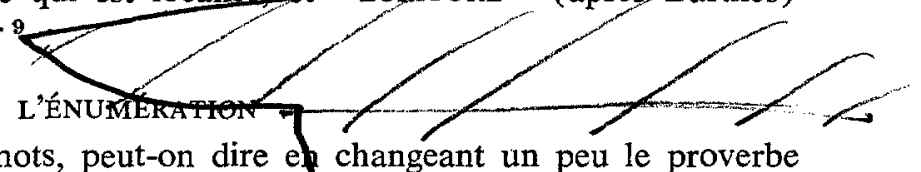

Un exemple vaut mille mots, peut-on dire ef changeant un peu le proverbe chinois; je choisis de prendre celui de l'énumération.

L'énumération est un type particulier développement du syntagme. Dans ce cas, une des branches est multiple au lieu d'être simple, Soit le syntagme

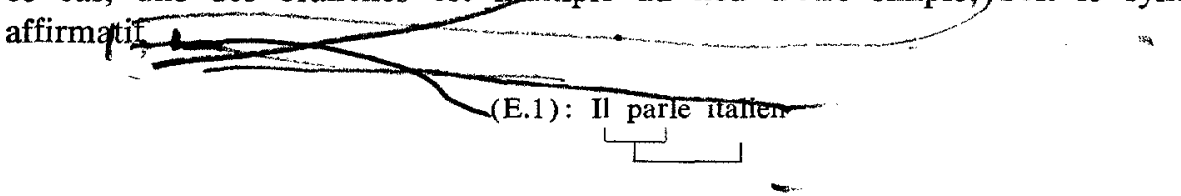

Il s'agit là d'un syntagme binaire habituel, du type AxcZ, dont on peut « ouvrir " à volonté l'un des termes: on obtient alors une énumération, au prix d'une explicitation du pronom de conjugaison. Ce faisant, on déplace l'accent d'insistance, tantôt sur A, tantôt sur Z:

(E.2): Jean, Jeanne, Lucie, Philippe, Anatole (et) Alphonse parlent italien

(E.3): Il parle italien, espagnol, anglais, français (et) chinois

Le fait que l'on peut «ouvrir» ainsi une des branches du syntagme est une donnée du système linguistique français. Cela relève donc de la linguistique

9. Depuis R. Barthes, le terme "écriture » a acquis une certaine caution et on le trouve un peu partout, notamment chez les critiques littéraires. Je l'ai relevé récemment dans le Devoir, à propos de Réal Benoit: "Votre écriture est-elle aussi onirique dans la Saison des artichauts qu'elle l'étaít dans Quelqu'un pour m'écouter? - L'écriture est plus simple, mais tout aussi onirique, je crois. $>$ (Le Devoir, 20 mai 1968.) 
et comme tel ne nous intéresse pas ici: du moins est-ce un élément négatif de la discussion. Disons que nous constatons qu'en français il existe un type de syntagme « énumératif». Dans la mesure où ce syntagme existe aussi en anglais, il ne donnera lieu à aucune difficulté particulière de traduction.

\section{L'ÉNUMÉRATION, PROCÉDÉ STYLISTIQUe}

Par contre, si nous considérons un grand nombre d'énumérations relevées dans des œuvres littéraires, nous serons amené à conclure qu'il en est de plusieurs sortes. C'est ici que nous touchons à la trilogie stylistique-style-écriture évoquée plus haut. La pluralité des types d'énumération, que nous allons essayer de préciser, peut se schématiser comme suit:

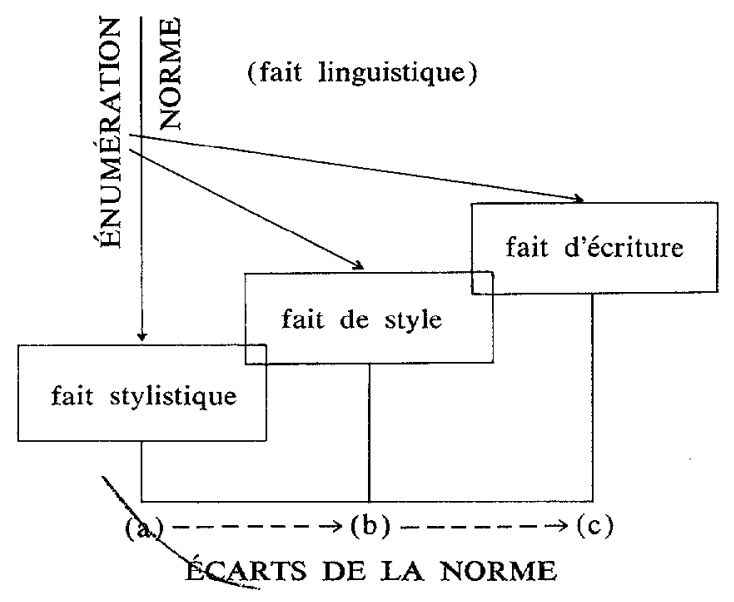

Nous allons constater que l'énumération, sur le plan stylistique, commun par conséquent à tous les locuteurs d'un moment donné de la langue, est un phénomène stylistique dont l'explication est peut-être à rechercher dans cette zone mixte, objet de la psycho-linguistique.

La question est de savoir ce qui transforme (E.2) et (E.3) en variantes stylistiques de (E.1). L'un des points qu'il faut constater dès l'abord est la présence ou l'absence de la conjonction et en (E.2) et (E.3): fait-elle partie du système, est-elle facultative, ou est-elle appelée par des ressorts stylistiques (charnière de terminaison) ? De plus, il serait important de savoir combien de termes on peut ajouter à l'une des branches du syntagme avant que le message ne devienne stylistique, c'est-à-dire avant que l'attention de l'auditeur se porte, non plus sur le contenu sémantique de l'énumération, mais sur son type syntaxique. Par exemple

(E.4): Mettez mon chapeau, mon pardessus et mes claques dans le vestiaire.

est une énumération «neutre» à laquelle on ne s'arrête pas, en principe. C'est aussi le cas de listes de termes (qui ne sont d'ailleurs pas des syntagmes), telles que les cotes de la Bourse, les résultats sportifs lus à la radio, le palmarès d'un concours, l'horaire du chemin de fer, l'annuaire du téléphone, etc. Ces énumérations sont «neutres» parce qu'elles ne sont pas structurées; il est donc difficile — et de peu d'intérêt - d'en prévoir la fin. Elles deviennent très vite monotones, 
l'intérêt fléchit et la mémoire a du mal à retenir ces éléments discontinus.

Mais dès que l'énumération a) entre dans un syntagme et' b) dépasse une certaine dimension, il s'établit un décalage entre la réalité et le message. Ce décalage, d'abord objet de surprise ou de malaise, déclenche en général le rire chez les auditeurs ou les lecteurs. On prête dès lors attention au jeu et non plus au sens; en fait, personne ne demande autant de précisions !

(E.5): Il se débarasse à la hâte de son chapeau, son manteau, son veston, ses claques, ses lunettes, son gilet, sa perruque et ses fausses dents.

L'exemple (E.5) rentre, me semble-t-il, dans la stylistique; sa longueur est inusitée, et peut-être certains éléments inattendus de l'énumération contribuent-ils aussi au caractère stylistique de l'exemple (perruque, fausses dents, claques pour des Français). D'ailleurs, l'énumération stylistique ne suscite pas que le rire: elle connaît un ton noble, mesuré, qui crée un temps de repos dans le déroulement du récit, comme le montrent les deux exemples suivants, empruntés à Robert Desnos:

(E.6): Le soleil rayonne, la rivière coule, à une fenêtre frémit le pot de géranium, une voiture passe sur l'autre rive...

(E.7): Mais sur la table on aperçoit le reflet, le reflet du jour sur un fruit et une bouteille, sur une assiette de faïence et sur un meuble...

\section{L'ÉNUMÉration, PROCÉdÉ de STYLE}

À partir du moment où ce procédé est exploité systématiquement dans le cadre de la création littéraire, il rentre dans l'arsenal des procédés de style et comme tel se retrouve à toutes les époques, chez des écrivains très divers qui s'en servent en général pour mettre en relief l'exagération (cocasse ou amère), l'enflure, et qui en espèrent un déclenchement comique. Leo Spitzer cite à ce propos les imprécations de Rabelais contre ses adversaires de la Sorbonne, qui sont à la fois un exemple de variation sur le mot Sorbonne et une énumération comique (cumul de deux procédés de style):

(E.8): Allez-donc, sophistes, sorbillans, sorbonagres, sorbonigènes, sorbonicoles, sorboniformes, sorbonisèques, niborcisans, sorbonisans, saniborsans... 10

1 La preuve que nous sommes dans le domaine du style, il faut la chercher dans les nombreux exemples des énumérations comiques, truc maintes fois repris aux époques classiques et romantiques ${ }^{11}$. On peut alors déceler à l'intérieur du genre des variations temporelles qui permettront de différencier nettement entre les exemples (E.9) et (E.10) ci-dessous:

(E.9) : Clindor. - Ce fer a trop de quoy dompter leur violence ...

Matamore. - Ouy, mais les feux qu'il jette en sortant de prison

Auroient en un moment embrazé la maison,

10. A propos de ces inventions verbales, qui sont, elles aussi, un procédé de style, Leo Spitzer note: "These word-families, representative of gruesome fantasy-beings ... have reality only in the world of language, established in an intermediate world between reality and irreality, between the nowhere that frightens and the here that reassures..." (Linguistics \& Literary History. Essays in Stylistics, Princeton, N.J., Princeton University Press, 1948, chap. 1, p. 16-17.) Noter que dans la mesure où ces termes sont inventés par Rabelais et lui sont particuliers, ils font partie de son écriture.

11. Cf. R. Garapon, la Fantaisie verbale et le comique dans le théâtre français. Le texte cité est pris dans l'édition Garapon; Société des textes français modernes, Paris, Didier, 1957. 
Dévoré tout à l'heure ardoises et goutieres,

Faites, lattes, chevrons, montants, courbes, fillieres,

Entretoises, sommiers, colomnes, soliveaux,

Parnes, soles, appuis, jambages, travetaux,

Portes, grilles, verroux, serrures, thuilles, pierre,

Plomb, fer, plastre, cimen, peinture, marbre, verre,

Caves, puits, cours, perrons, salles, chambres, greniers,

Offices, cabinets, terrasses, escaliers:

Juge un peu quel désordre aux yeux de ma charmeuse !

(Pierre Corneille, l'lllusion comique (1636), acte III, scène v.)

(E.10): Comme j'étais assez près d'arriver [à la ville], je vis sortir des murailles en grande pompe un cortège innombrable qui faisait route de mon côté, et je reconnus sur-le-champ l'objet de cette excursion scientifique à l'attirail des voyageurs. C'étaient des planches, des perches, des échelles, des cordes, des poulies, des barres, des leviers, des poids, des contrepoids, des roues, des cabestans, des moufles, des grues, des dragues, des griffes, des grappes, des tracs, des pics, des crocs, des crics, et tout le mobilier du Conservatoire des Arts \& Métiers, à l'exception d'une bascule. (Charles Nodier, Hurlubleu, 1830.)

Ces quelques exemples doivent suffire pour établir le bien-fondé de la catégorie énumération parmi les procédés de style du français littéraire aux différentes époques. Il faut maintenant savoir si l'anglais connaît ce même procédé, si l'écart entre l'énumération linguistique et l'énumération stylistique y est également visible, de façon à prévoir les modalités de traduction d'une langue à l'autre. Je laisse aux spécialistes de la stylistique anglaise le soin de décider si les deux langues réagissent de la même façon à ce procédé. Qu'il suffise d'en donner ici un exemple; je le choisis dans un texte de Thomas Nash (The Unfortunate Traveller) dont les éditions Aubier ont donné récemment une traduction juxtalinéaire:

(E.11): That daie come, flourishing entred John Leiden the butcher into the field, with a scarfe made of lists, [...] his men were all base handies craftes, as cobblers, and curriers, and tinkers, whereof some had barres of yron, some hatchets, some coole staves, some dung forks, some spades, some mattocks, some wood knives, some addises for theyr weapons... (Thomas Nash, The Unfortunate Traveller, London, The Verona Society, 1930 (1594), facsimile edition.)

\section{L'ÉNUMÉRATION, PROCÉDÉ D’ÉCRITURE}

Passons sans transition à l'écart maximum évoqué dans le schéma en considérant l'énumération comme une idiosyncrasie personnelle de certains auteurs. En fait, ces considérations s'appliquent aussi aux exemples ci-dessus, comme nous l'avions remarqué pour le texte de Rabelais. Dans l'ensemble des procédés stylistiques, les niveaux se chevauchent et ce n'est qu'au prix d'une certaine artificialité que le stylisticien réussit à les maintenir séparés.

Là où la situation se complique, c'est lorsque les procédés de style devenant personnels, relevant par conséquent de l'écriture, trahissant la psychologie profonde de l'auteur, exigent du traducteur un effort semblable pour évoquer un personnage tout autant qu'un texte. C'est le cas du texte (E.12) que j'emprunte à Jacques Perret. Ce dernier est féru d'énumérations, qu'il enjolive (comme Rabelais) d'inventions verbales cocasses, accumulant ainsi les effets au risque de fatiguer le lecteur pressé d'arriver aux conclusions. L'énumération entre donc dans la structure profonde du moi de Jacques Perret, et il faudra que le traducteur rende en anglais tous les écarts qui trahissent cette structure, c'est-à-dire transpose, module, cherche des équivalences, bref fasse illusion. L'étude d'écrivains de même personnalité (James Joyce?) devrait fournir de bons exemples anglais équivalents. 
(E.12): [Il s'agit d'un palan nouveau que Perret et son ami cherchent à inventer pour leur bateau de plaisance, et qui devrait évidemment servir à tout ...] A vrai dire, l'idée d'un palan à tout faire frappé sur le grand mât était un vieux projet maintes fois évoqué. Dans notre idée ce palan polyvalent, ce polypalan, devait, bien entendu, parer à toute avarie de drisse, mais Collot y prévoyait aussi le moyen de ne pas abandonner dans le sillage le phoque ou le lamentin que nous aurions eu l'occasion de harponner. Nous pensions également aux tortues de rencontre, aux baleineaux éventuels, aux requins de fortune, aux coelacanthes arriérés, aux pieuvres ampoulées, au cachallopathe isthmicole, au grand silure hellespontin, au macrosaure inqualifiable, à tous les monstres enfin de la génétique officielle ou romanesque, sauf bien entendu la baleine blanche... Dans l'intervalle de ces captures, le croc palanqué avait pour mission d'amener à bord toutes sortes d'épaves telles que baril, coffre, balle d'indigo, caisse d'épices, cochenilles en poudingue, vieille peau de sirène en mue, carlingue d'avions foudroyés, bouteille vide ou manuscrit en bonbonne, valise diplomatique, ambre gris, boîte de ration $K$, bâton flottant, cage à poules, cantine d'officier supérieur, ceufs d'oiseau biligou, châsse de saint breton, noyé de haut lignage, écume de pipe amère, chapiteau de pierre ponce noyé de l'atlantide, vistamboir hydrofuge, dame-jeanne de tafia, piano à queue ... (Jacques Perret, Rôle de Plaisance, N.R.F.)

Le lecteur aura relevé tous les termes issus de l'imagination débordante de l'auteur, tels que vistamboir, cachallopathe, polypalan, ainsi que les syntagmes associatifs « raisonnables» tels que écume de pipe amère (cf. écume de mer), cochenilles en poudingue, qui devront être créés parallèlement par le traducteur, à partir des ressources et des associations de l'anglais. Nous touchons là aux limites de la traduction, pour entrer dans le domaine de la création littéraire. C'est déjà de la poésie; cela demande un art achevé. Il faut un Jacques Perret anglais pour traduire Jacques Perret, et toutes les réussites peuvent alors se discuter. En matière d'écriture, traduire est donc un art, si l'on veut que le lecteur retrouve dans le nouveau texte quelque chose du frisson de l'original.

C'est là le plus haut défi lancé aux traducteurs.

\section{APPENDICE BIBLIOGRAPHIQUE}

L'univers peut être exploré avec nos seules ressources, à condition de concentrer suffisamment d'attention sur les points vraiment pertinents. Je ne sache pas qu'Einstein ait trouvé sa formule de la Relativité en feuilletant les pages roses du Petit Larousse.

Cependant, et à condition de ne, pas s'alourdir de démarches non essentielles, il est bon de connaître ce que d'autres ont écrit sur le sujet qui nous intéresse; une consultation de leurs ouvrages ouvre l'esprit à celui qui sait ce qu'il veut y trouver.

C'est pourquoi je me suis permis de terminer ces brèves remarques sur la traduction littéraire par l'indication de quelques écrits rencontrés récemment aux détours de la route, à l'heure de la lecture de minuit " pour se faire escholier". Bien sûr, un autre lecteur aurait feuilleté pour vous d'autres pages: la bibliographie du style est très vaste et mal organisée. Elle mériterait une publication séparée. A condition de s'en tenir aux travaux qui touchent directement le traducteur, ce serait là ceuvre utile que META pourrait entreprendre pour le plus grand profit de ses lecteurs. A ce sujet, le mémoire de $M^{\text {le }} \mathrm{G}$. Gagnon (cf. $\mathrm{n}^{\circ}$ 5.4) contient déjà le noyau d'une telle bibliographie (p. I-XXX) ainsi que l'important cours de stylistique française donné par elle à la télévision. On pourrait demander à l'auteur qu'elle « édite " son texte en l'orientant plus particulièrement vers les problèmes de TL.

\section{Sources}

1.1 Varia: excellents articles parus récemment dans les principales revues de linguistique et de stylistique: Diogène, Babel, Lingua e stile, Word, Linguistics, le Français moderne, Der Uebersetzer, Traduire; consulter 1.2 et 1.3 .

1.2 Index translationum, répertoire multilingue des traductions, Paris, U.N.E.S.C.O. [annuel].

1.3 Bulletin signalétique du C.N.R.S., $3^{\mathrm{e}}$ partie: Philosophie et sciences humaines; $\mathrm{n}^{\circ} 19$ : Problèmes de la traduction, Paris, Centre national de la recherche scientifique [semes-
triel].

1.4 Une très bonne bibliographie se trouve dans E. CARY et R.W. JUMPELT, la Qualité en matière de traduction / Quality in Translation, Actes du III' Congrès international de la 
F.I.T., Oxford, Pergamon Press, 1963. [R.W. Jumpelt continue, de pair avec R. Haeseryn, ses notes bibliographiques dans Babel.]

1.5 HATZFeld, $\mathrm{H}$. et Y. LE HrR, Essai de bibliographie critique de stylistique française et romane, 1955-1960, Paris, Presses Universitaires de France, 1961.

1.6 Horguelin, Paul A. et al., "Les outils du traducteur - Bibliographie ", META [passim]; notamment XII, 3 (1967): 98-101. Les livres importants sont accompagnés d'un commentaire de présentation.

1.7 Ullmann, Stephen, "Some General Works on Style ", Style in the French Novel, cf. 4.11.

\section{DÉfinitions}

2.1 Delboutle, P., "Définition du fait de style ", Cahiers d'analyse textuelle, II (1960): 94-104.

2.2 Enkvist, N.E. (ed.), Linguistics and Style, London, Oxford University Press, 1964.

2.3 Fowler, Roger, "Linguistics, Stylistics, Criticism? ", Lingua, XVI, 2 (1966): 153-165.

2.4 LE BiDoIs, Robert, "Défense et illustration de la traduction " [rubrique: Défense de la langue française], le Monde, 27 mars 1968.

2.5 IMBS, P., "Analyse linguistique, analyse philologique, analyse stylistique ", Programme du Centre de philologie romane, Strasbourg (1957): 61-79.

2.6 SAYCE, R.A., "The Definition of the Term Style ", Proceedings of the Third Congress of the International Comparative Literature Association (1962): 156, 166.

2.7 Sebeok, Thomas A. (ed.), Style in Language, New York, John Wiley \& Sons, 1960. [Voir en particulier l'article de Roman Jakobson, "Linguistics and Poetics ».]

2.8 Sempoux, A., "Trois principes fondamentaux de l'analyse du style ", Revue belge de philologie et d'histoire, XXXVIII (1960): 809-814.

2.9 SEMpoux, A., "Notes sur l'histoire des mots style et stylistique", Revue belge de philologie et d'histoire, XXXIX (1961): 736-746.

\section{3. Évaluation}

3.1 Carrol, John B., * An Experiment in Evaluating the Quality of Translations ", Mechanical Translation, IX, 314 (1966): 55-66.

3.2 Mrtler, G.A. and J.G. Beebe-Center, \& Some Psychological Methods for Evaluating the Quality of Translations ", Mechanical Translation, 3 (1958): 73-80.

3.3 U.S. GOVERNMENT, Machine and Thought: Excerpts (Evaluation of Ouality in Translating), U.S. Dept. of Commerce, Office of Technical Services, TT65-60307, Washington, D.C., 1965.

3.4 [Voir aussi Cary et Jumpelt, $\mathrm{n}^{\circ} 1.4$ ]

3.5 URE, Jean and Jeffrey ElLIS, An Exercise in the Use of Descriptive Linguistic Techniques in Literary Translation, pre-publication, Edinburgh, 1966.

\section{OUVRAGES GÉNÉRAUX 12}

4.1 CARY, Edmond, les Grands Traducteurs français, Genève, Georg, 1963.

4.2 Fowler, Roger (ed.), Essays on Style and Language, London, Oxford University Press, 1966.

4.3 Godn, Henri J.G., les Ressources stylistiques du françis contemporain, Oxford, Blackwell, 1948. [Utilise des citations littéraires et discute avec perínence 12 textes littéraires traduits en anglais.]

4.4 Joos, M. The Five Clocks. A Linguistic Excursion into the Five Styles of English Usage, New York, Harcourt Brace, 1961. [Voir l'important compte rendu que J. Darbelnet a consacré à ce livre, University of Toronto Quarterly (July 1968): 451-455.]

4.5 Levin, Samuel P., Linguistic Structures in Poetry, La Haye, Mouton, 1962.

4.6 Mounin, Georges, les Belles Infidèles, Paris, Cahiers du Sud, 1955.

4.7 Segger, S.W., Thèmes modèles, Cambridge University Press, 1964. [Le mot thème est vectoriel; il désigne une traduction faite vers la langue seconde, et non dans la langue maternelle (version); ce qui est version pour les francophones est donc thème pour les

12. Il faudrait ajouter à cette liste des ouvrages très récents ou en cours d'impression qui pourront utilement préciser certains des points soulevés dans cet article. Je songe notamment à Bernard Dupriez, Traité de stylistique littéraire (thèse de doctorat, Université de Strasbourg), ainsi qu'aux articles de la nouvelle revue américaine Language and Style (Southern Illinois University). 
anglophones, et vice versa. On trouvera, outre d'utiles conseils, 85 traductions littéraires de textes anglais en français, dans la grande tradition de Ritchie et Moore.]

4.8 StéPaNoff, Georges, Francuzskaia Stilistika, Moscou, "Vyschaia chkola ", 196513.

4.9 MacAndrew, R., " The Hazardous Art of Mistranslation », Harper's Magazine, April 1966.

4.10 Compte rendu d'Incubation de G. Bessette, traduit par G. Shortliffe, Canadian Literature (Vancouver), 36 (1968).

4.11 Ullmann, Stephen, Style in the French Novel, Oxford, Blackwell, 1964.

\section{5. ÉTUdES PARTICULIÈrES}

5.1 AHrweIler, Alice, "Réalisme et traduction ", Europe, 82 (1952). [Sur la traduction poétique.]

5.2 Des MarchaIs, Gilles, la Grammacritique, Montréal, Leméac, 1965.

5.3 EtkInd, Efim, «La stylistique comparée, base de l'art de traduire», Babel, XIII, 1 (1967): 23-30. [Il s'agit de traduction littéraire.]

5.4 Gagnon, Gilberte, Conceptions contemporaines de la stylistique, mémoire de D.E.S., Université de Montréal, Département de linguistique, 1966, XXV-132 p., polycopié.

5.5 Hatzfeld, H., "Peut-on systématiser l'analyse stylistique ? ", Rivista di letterature moderne, XIII (1960): 149-157.

5.6 Koszul, A., "A propos d'une nouvelle version des Sonnets de Shakespeare 》 et J. LorSEAU, "A propos d'une nouvelle édition avec traduction de Shakespeare", Etudes anglaises, IX, 1 (1956): 1-13.

5.7 Matrioli, E., "Introduzione al problema del tradurre », Arte, critica, filosofia, Bologna, Patron (1966): 189-214.

5.8 OMMANN, Richard, "Generative Grammar and the Concept of Literary Style ", Word, XX (1964): 423-439.

5.9 Rossr, A., "Traduzione e evoluzione letteraria ", Paragone, 186 (1965).

5.10 Vinay, J.-P., " Trio pour deux musiciens, ou Comment définir la stylistique ? ", University of Toronto, School of Graduate Studies, July 1967.

JEAN-PAUL VINAY

13. Cette référence n'est pas uniquement de la poudre aux yeux. Je sais bien que peu nombreux sont les traducteurs qui peuvent jongler avec les déclinaisons russes ou même simplement déchiffrer l'alphabet cyrillique. Mais la tradition de "stylistique comparée " est très solidement ancrée en U.R.S.S.; d'après les travaux que nous en recevons, il est évident que tout ce qui est publié en Europe occidentale et au Nouveau Monde est suivi de très près, dépouillé et commenté. C'est ainsi que les pages du traité de Georges Stépanoff abondent en observations pertinentes sur les ressources stylistiques du français, vues dans l'optique du traducteur russe. Les exemples qu'il donne, riches et variés, permettent à eux seuls de s'en rendre compte, ainsi que la liste des ouvrages cités. Il appartient, me semble-t-il, à nos collègues spécialistes des langues slaves, de nous renseigner régulièrement sur cette production abondante et stimulante. C'est ce qu'a fait pour le Journal des Traducteurs à plusieurs reprises W. Grebenschikov (IX, 3 (1964): 75). 